\title{
CARACTERÍSTICAS DE LOS ALIMENTOS Y CONTROL DE CALIDAD
}

\author{
DRA.ALEJANDRINA GALLEGO PICÓ \\ Departamento de Ciencias Analíticas. \\ Facultad de Ciencias. UNED
}

\section{CARACTERÍSTICAS DE LOS ALIMENTOS}

Todos los seres vivos necesitan alimentarse para reponer las pérdidas de materia consumida por la actividad del organismo, que permitan producir las sustancias necesarias para la formación de nuevos tejidos, favoreciendo el crecimiento y para transformar la energía contenida en los alimentos en calor, movimiento y trabajo.

Por consiguiente, los alimentos son productos, naturales o elaborados, que toman los seres vivos y que proporcionan al organismo las sustancias nutritivas y la energía que necesitan para vivir. Estas sustancias nutritivas o nutrientes son las sustancias químicas vitales para el crecimiento y mantenimiento del organismo. Como ya se ha mencionado aportan energía, forman y reparan las estructuras corporales y regulan los procesos metabólicos.

Las características de los alimentos la podemos abordar desde diferentes perspectivas. Desde el punto de vista de su composición físico-química, es decir que compuestos químicos constituyen los alimentos pero también se puede abordar desde las características nutricionales que tienen los mismos. En este apartado se incidirá exclusivamente en los aspectos físico-químicos de los alimentos, aunque también revisaremos algunas de sus características nutricional.

Desde el punto de vista físico-químico, los alimentos se componen principalmente de compuestos orgánicos: hidratos de carbono, lípidos y proteínas y también de otros inorgánicos como es el agua, o los metales.

\section{Hidratos de carbono}

Bajo la denominación de hidratos de carbono o glúcidos se reúnen los azúca- 
res y las sustancias derivadas directamente de ellas. Su fórmula empírica general es $\mathrm{C}_{\mathrm{n}} \mathrm{H}_{2 \mathrm{n}} \mathrm{O}_{\mathrm{n}}$, pero sin olvidar que no todos los compuestos con esta fórmula son necesariamente hidratos de carbono (por ejemplo, el ácido láctico es $\mathrm{C}_{3} \mathrm{H}_{6} \mathrm{O}_{3}$ ).

Los hidratos de carbono tienen una gran importancia biológica, pues son la puerta de entrada al mundo orgánico, siendo los primeros que se sintetizaron. Además su función en la naturaleza es muy variada. Forman parte de la estructura de los seres vivos (celulosa, quitina). También constituyen sustancias de reserva (almidón, glucógeno). Desde el punto de vista metabólico son los primeros en quemarse antes que los lípidos y las proteínas.

Los hidratos de carbono más sencillos son los monosacáridos. Éstos son azúcares fundamentales de 3 a 9 átomos de carbono. Desde el punto de vista químico son alcoholes polivalentes con un grupo aldehídico (aldosas) o cetónico (cetosas). Los monosacáridos son dulces, solubles en agua, cristalizables y presentan actividad óptica. Son monosacáridos la glucosa, que se encuentra en muchas frutas, en la miel o en la sangre; la fructosa que se presenta asociada a la anterior en frutas y miel, o la galactosa, que aunque no se encuentra libre forma parte de otros hidratos de carbono más complejos.

Los disacáridos, formados por condensación de dos moléculas de disacáridos son igualmente importantes, entre ellos se encuentra la sacarosa que es el azúcar común, la lactosa, que es el azúcar de la leche, la maltosa o azúcar de malta o la celobiosa que aunque no se encuentra libre es precursora de la celulosa.

Los hidratos de carbonos más complejos se dividen en dos grandes grupos, los glucanos que son homopolimeros de monosacáridos y los heteropolisacáridos que cuentan en su molécula con un monosacárido y otro grupo.

A diferencia de los monosacáridos y disacáridos los glucanos no son dulces y al tener un alto peso molecular no son muy solubles en agua, tampoco cristalizan y suelen formar disoluciones coloidales. Son glucanos el almidón que se encuentra en los órganos de reserva de las plantas, el glucógeno o almidón animal que se encuentra en el hígado y en los músculos y también la celulosa.

Algunos de los heteropolisacáridos más importantes son: la quitina que se encuentra en las algas, crustáceos y moluscos; la lignina de la madera, la hemicelulosa que se localiza también en las plantas. Ésta se encuentra asociada a las pectinas, otro heteropolisacárido derivado del ácido galacturónico, y celulosas formado las paredes vegetales. Los carragenos son un grupo de heteropolisacáridos sulfatados entre los que se encuentra la carragina o el agar-agar. También pertenecen a este grupo 
los mucopolisacáridos, éstos compuestos son viscosos, de ahí su nombre. El ácido hialurónico y la heparina pertenecen a este grupo.

Desde el punto de vista de la industria agroalimentaria los hidratos de carbono tienen múltiples aplicaciones debido a:

\section{Poder edulcorante}

Todos los azúcares (monosacáridos y disacáridos) poseen cierto sabor dulce relacionado con la presencia de grupos hidroxilo de su molécula, su estereoquímica y la posibilidad de formar puentes de hidrógeno.

El poder edulcorante depende de que azúcar se trate, de su configuración alfa o beta, de la temperatura y de la concentración. La -manosa constituye una excepción pues tiene un sabor amargo.

\section{Conservante}

Los azúcares se han utilizado como conservante en la preparación de mermeladas, leche condensada. El poder conservante se fundamenta en el aumento de la presión osmótica en el medio, deshidratando los microorganismos e inhibiendo su crecimiento, y por tanto, eliminándolos.

\section{Espesante}

Los almidones, la celulosa o la carragenina se utilizan frecuentemente como espesantes en la industria alimentaria.

\section{Gelificante y emulsionante}

Las pectinas en presencia de sacarosa presentan propiedades gelificante siendo utilizadas en la fabricación de mermeladas y productos similares. También los almidones y las carrageninas o el agar-agar son utilizados con estos fines.

\section{Suspensión}

Las pectinas también se emplean en la industria farmacéutica para preparar suspensiones de los medicamentos. Por otra parte, las propiedades coloidales de las pectinas permiten tener en suspensión los sólidos de los zumos de fruta naturales que pueden ser precipitados por enzimas pépticas.

\section{Floculante}

Algunos hidratos de carbono como la carragenina es utilizada para la clarificación de la cerveza. 


\section{Control de la cristalización}

Como se ha mencionado los azúcares son fácilmente cristalizables, sin embargo esta característica puede no ser deseable en determinados productos y debe controlarse. Así por ejemplo, la glucosa líquida inhibe la cristalización de la lactosa de la leche evitando una textura terrosa. En la preparación de la leche en polvo se controla la cristalización para inducir la cristalización en la forma beta que es más soluble que alfa. También la celulosa es utilizada se utiliza en helados y otros congelados para reducir los daños estructurales causados por congelación.

\section{Humectante}

Algunos azúcares son higroscópicos. Esto no es deseable en la leche en polvo ni en los productos granulados. La mezcla de alfa y beta lactosa reduce el poder higroscópico de éstos preservando el producto. En otros casos, interesa mantener cierta humedad constante en el producto para ello se utilizan algunos carbohidratos de alto peso molecular que absorben el agua y la fijan. También la celulosa se utiliza como humectante en pudines y flanes para impedir la sinéresis de los mismos.

\section{Color y sabor}

La caramelización, es decir someter a alta temperatura los azúcares da sabor y color. Los azúcares en presencia de calor reaccionan con las proteínas ( $\mathrm{R}$. Maillard) dando un color y sabor característico a los alimentos (panificación, tostado de café, etc.). También algunos azúcares, como la lactosa, presentan un poder adsorbente y es utilizada como agente base para retener sabores artificiales, aromas y colores.

Otras aplicaciones. Los hidratos de carbono son también utilizados como adhesivos (goma arábiga o almidón), para fabricar películas resistentes que permiten elaborar cápsulas terapéuticas (carragenina). Como medios de cultivos microbiológicos (agar o hidrolizados de celulosa), etc.

\section{Lípidos}

Los lípidos son un grupo de compuestos cuya estructura química no presenta mucha similitud, pero todos ellos tienen en común que son solubles en disolventes orgánicos (éter, cloroformo, acetona, etc.) e insolubles en agua. Se les ha clasificado en tres grandes grupos: lípidos simples, lípidos compuestos y compuestos asociados. 
Lípidos simples

Los lípidos simples químicamente son ésteres de ácidos grasos y alcoholes. Las grasas, aceites y las ceras pertenecen a este grupo. Las grasas y aceites son ésteres de ácidos monocarboxílicos y glicerina (trialcohol) mientras que las ceras son ésteres de ácidos grasos y alcoholes monohidroxilados. Las diferentes grasas y ceras están caracterizadas por los ácidos grasos que las forman.

\section{Lípidos compuestos}

Los lípidos compuestos son lípidos simples conjugados con moléculas no lipídicas. Dentro de este grupo estarían los fosfolípidos, glucolípidos y lipoproteínas. Los fosfolípidos son lípidos en cuya composición entra el ácido ortofosfórico y una base orgánica nitrogenada. El más importante es la lecitina que se presenta en un 10\% en la yema de huevo. Los fosfoglicéridos tienen una gran importancia biológica, pues intervienen en distintas fases del metabolismo y son parte integral de las membranas celulares. Los glucolípidos y lipoproteínas son compuestos, como su nombre indica, de lípidos e hidratos de carbono, en el primer caso y proteínas en el segundo. Del primer grupo los más importantes son los cerebrósidos y del segundo la lipovitelina que se encuentra en el huevo.

\section{Compuestos asociados}

En este grupo se incluyen los carotenoides y los esteroides. Los carotenoides son pigmentos amarillos anaranjados o rojos que se encuentran abundantemente en la naturaleza. La carotina de color naranja da color a la zanahoria, pimiento y tomate. Un derivado de la carotina es la vitamina A. También es un carotenoides la xantofila que da la mayor parte de los colores amarillos a las plantas o la crocetina del azafrán.

Los esteroides son un importante grupo de sustancia que incluyen desde el colesterol hasta vitaminas y hormonas. La ergosterina (provitamina $\mathrm{D}_{2}$ ), el 7dehidrocolesterol (provitamina $\mathrm{D}_{3}$ ), las hormonas sexuales (testosterona $\mathrm{y}$ progesterona), las hormonas de la corteza suprarrenal (cortisona), los ácidos biliares, la digitalina, y otros muchos.

Debido a la heterogeneidad y características de los lípidos, estos presentan múltiples funciones:

Función de reserva

Son la principal reserva energética del organismo. Un gramo de grasa pro- 
duce 9'4 $\mathrm{kcal} / \mathrm{g}$ en las reacciones metabólicas de oxidación, mientras que proteínas y glúcidos sólo producen 4’1 kcal/g.

\section{Función estructural}

Forman parte de las membranas celulares. Recubren órganos y le dan consistencia, o protegen mecánicamente o térmicamente (tejido adiposo, ceras)

\section{Función biocatalizadora}

Favorecen o facilitan las reacciones químicas que se producen en los seres vivos. Cumplen esta función las vitaminas lipídicas y las hormonas esteroideas.

\section{Función transportadora}

El transporte de lípidos desde el intestino hasta su lugar de destino se realiza mediante su emulsión gracias a los ácidos biliares y colesterol

Respecto a la industria agroalimentaria, las grasas y aceites son el grupo más importantes. Sus características y su deterioro tienen gran influencia en la tecnología de los alimentos. Por ejemplo, durante su almacenamiento, las grasas sufren cambios en sus formas cristalinas, lo que trae consigo modificaciones en sus puntos de fusión, afectando a algunas aplicaciones industriales. La manteca de cacao presenta un alto grado de polimorfismo que afecta considerablemente la elaboración de chocolates. La rancidez y la reversión son otros ejemplos de deterioro de las grasas con importante repercusión en la elaboración de alimentos. Las grasas y los aceites sufren con el paso del tiempo deterioro en su valor nutritivo y presentan olor y sabor desagradables. Este hecho puede explicarse químicamente debido a la hidrólisis química o enzimática del enlace éster, y a que los ácidos grasaos insaturados son sensibles a reacciones de oxidación. Este proceso se conoce como rancidez o enranciamiento de las grasas y aceites. El grado de rancidez dependerá del tipo de grasa o aceites. La reversión se produce durante el almacenamiento de los aceites y da lugar a la presencia de olores desagradables producidos por la formación de derivados aldehídicos y cetónicos del ácido linoléico principalmente. Otra problemática ligada a las grasas su posible toxicidad. Las grasas al freír alcanzan temperaturas de hasta $190^{\circ} \mathrm{C}$, lo que favorece la captación de agua del aire que le rodea. En estas condiciones se facilitan las reacciones de oxidación y la formación de compuestos potencialmente 
tóxicos. El calentamiento de ácidos grasos insaturados produce compuestos aromáticos policíclicos potencialmente cancerígenos. Existe una relación directa entre el grado de instauración de la grasa y la producción de dichos compuestos. En la actualidad se piensa que los peróxidos no son tan tóxicos como los productos secundarios provenientes de su degradación. Los peróxidos además pueden destruir, debido a su reactividad, vitaminas y proteínas.

\section{Proteínas}

Otro de los grandes grupos que componen los alimentos son las proteínas. Las proteínas son polímeros de aminoácidos, es decir cadenas carbonadas con un grupo amino y con un grupo carbonilo. Las proteínas son específicas de cada especie. Todas las especies son capaces de sintetizar aminoácidos pero algunos de los aminoácidos deben ser adquiridos a través de la alimentación. Hay 8 aminoácidos que el organismo humano no puede sintetizar y que son indispensables para su dieta, son los llamados aminoácidos esenciales: valina, leucina, isoleucina, treonina, metionina, fenilalanina, triptófano y lisina. Las proteínas se pueden clasificar de muy diferentes formas. Respecto a su composición se clasifican en simples (albúminas, globulinas, protaminas, histonas, etc.) y conjugadas (metaloproteínas, glicoproteínas, lipoproteínas, nucleoproteínas, etc.).También se pueden clasificar según su estructura. Las cadenas peptídicas (estructura primaria) pueden formar una serie de hélices o cadenas (estructura secundaria) que luego se pueden enrollar (estructura terciaria), y posteriormente formar conglomerados (estructura cuaternaria).

Las proteínas están implicadas en importantes funciones biológicas que incluyen la desde la mera función estructural como es el caso del colágeno o la queratina, o también la miosina del músculo, hasta la función enzimática (lipasas, proteasas, catalasas, etc.) y hormonal (insulina, oxitocina,...). Son proteínas algunas toxinas (faloidina, toxina botulínica), los anticuerpos (seroglobulinas, fibrinógeno) y algunos de los compuestos que intervienen en el transporte de oxígeno (hemoglobina, mioglobina).

También tienen una gran importancia en la industria agroalimentaria como gelificantes, espumantes, emulsionantes o como responsables de color y sabor en algunos productos.

La gelatina, producida al cocer el tejido conjuntivo rico en colágeno (escleroproteína) es un conocido gelificantes. Las albúminas de la clara de hue- 
vo, al tener la capacidad de desnaturalizarse y formar espuma pueden ser utilizadas como espumantes. Las caseínas se utilizan como emulsionantes en productos cárnicos y embutidos, así como la lecitina o la lipovitelina. Como ya se ha mencionado al hablar de los hidratos de carbono, existen reacciones entre las proteínas y los azúcares (reacciones de Maillard) que dan color y sabor característicos a los alimentos. Por otra parte, las enzimas son proteínas de origen natural que catalizan las reacciones biológicas y se utilizan con frecuencia en la industria agroalimentaria. La amilasa en la malta germinada convierte el almidón en azucares fermentables por levaduras. La invertasa en la miel permite a las abejas transformar sacarosa en la mezcla fructosa glucosa. La lipasa hidroliza las grasas en los quesos para producir sabores característicos. La proteasa degrada el gluten de la harina de trigo y causa una reducción en el volumen del pan.

\section{Vitaminas y minerales}

Desde el punto de vista nutricional los componentes de los alimentos se pueden clasificar en macronutrientes y micronutrientes. Los macronutrientes son aquellos constituyentes que suministran la mayor parte de la energía metabólica al organismo. Los principales son los hidratos de carbono, los lípidos y las proteínas. Las vitaminas y los minerales son considerados micronutrientes su aporte es necesario en pequeñas dosis pero son imprescindibles para el correcto funcionamiento del organismo.

Las vitaminas comprenden un grupo diverso y heterogéneo de compuestos orgánicos que son, desde el punto de vista nutricional, micronutrientes esenciales imprescindibles para el organismo, necesarios para poder sintetizar otros nutrientes. Las vitaminas no generan energía, y sus carencias o deficiencias originan trastornos o patologías concretas.

La nomenclatura de las vitaminas siempre ha sido problemática, pues al principio de su descubrimiento era imposible la utilización de su nombre químico al desconocerse su estructura. Como consecuencia de ello, se empezó a utilizar una nomenclatura sistemática que consistía en asignar a cada vitamina que se descubría una letra del alfabeto, vitamina A, vitamina B, etc. Sin embargo, posteriormente también se tuvieron problemas en el momento que se descubrió que, por ejemplo, la vitamina $B$ era en realidad un conjunto de numerosas y diferentes vitaminas, por lo que la solución fue la utilización de subíndices, como B1, B2, B6, etc.

Actualmente, se utilizan nombres químicos bastante simples, aunque las 
primeras denominaciones aún continúan vigentes en muchas vitaminas, como por ejemplo en el caso de la vitamina $\mathrm{C}$ o ácido ascórbico.

Desde el punto de vista estructural las vitaminas no presentan nada en común y su función biológica tampoco facilita mucho su clasificación, por lo que el único criterio de clasificación que ha persistido es la división de las mismas en hidrosolubles (las más importantes son el ácido ascórbico o vitamina $\mathrm{C}$, las del grupo B y el ácido fólico), y liposolubles.

Los elementos $\mathrm{C}, \mathrm{H}, \mathrm{O}$ y N , son los constituyentes fundamentales del cuerpo humano, pero además, éste contiene y necesita una serie de elementos que están presentes en los alimentos, y que se ingieren como sales minerales, aunque en muchos casos forman parte de complejos orgánicos (como el caseinato cálcico de la leche).

Los elementos minerales se encuentran en los alimentos a concentraciones relativamente bajas, pero desempeñan papeles clave tanto en los sistemas vivos como en los alimentos. Estos elementos se encuentran en las cenizas de los alimentos y pueden analizarse en ellas, después de la incineración.

Existe un grupo de elementos minerales que están en mayor proporción en el organismo y se llaman elementos mayoritarios o macroelementos ( $\mathrm{Ca}, \mathrm{P}, \mathrm{K}, \mathrm{Cl}, \mathrm{Na}$, $\mathrm{Mg}, \mathrm{S})$, y otros que están en cantidades menores y se llaman elementos minoritarios o microelementos u oligoelementos (Fe, $\mathrm{Zn}, \mathrm{Cu}, \mathrm{Mn}, \mathrm{I}$, F, etc.). Finalmente, otros están en cantidades todavía mucho más pequeñas y se llaman elementos traza.

Por otro lado, se denomina elemento mineral esencial al elemento que su eliminación, de la dieta u otra ruta de exposición a un organismo, conduce a un deterioro constante y reproducible de una función fisiológica.

Los requerimientos humanos de minerales esenciales oscilan entre unos pocos microgramos diarios a un $1 \mathrm{~g} /$ día. Si la ingesta es baja durante un cierto tiempo, aparecerán los signos de carencia, e inversamente una ingesta demasiado elevada puede conducir a toxicidad.

En los alimentos se encuentran muchas formas químicas distintas de los minerales. Estas formas suelen denominarse especies e incluyen compuestos, complejos e iones libres.

Los elementos minerales abundan en casi todos los alimentos y, aunque su proporción puede variar según las zonas de producción y las condiciones de cultivo, sus carencias son raras. Durante la elaboración de materias primas, la mayoría de las veces, se producen cambios en el contenido en minerales, como por 
ejemplo en procesos térmicos y en separaciones. Asimismo, las pérdidas de nutrientes minerales en los procesos industriales o culinarios, por lavado, escaldado o cocción, son importantes.

\section{CONTROL DE CALIDAD}

En las sociedades desarrolladas, la demanda de los consumidores no sólo exige que los productos agroalimentarios cubran las necesidades nutricionales, sino que también sean apetitosos, saludables y seguros. Actualmente además, existe una gran sensibilidad por parte de los consumidores sobre el impacto de los procesos de fabricación y distribución en el medio ambiente, del trato y bienestar de los animales utilizados en la producción, e incluso de la aplicación correcta de prácticas agrícolas que pueden causar un impacto negativo en la cadena alimentaria o en el medio ambiente.

Esta nueva realidad ha llevado a la industria agroalimentaria ha cambiar su orientación e impulso, pasando de fijar objetivos centrados en la producción, a prestar atención preferente a las demandas del consumidor.

Los alimentos pueden ser descritos mediante una serie de parámetros o variables que se transforman en atributos por la percepción y preferencias del consumidor. Un atributo innegociable de cualquier producto es la calidad higiénica y sanitaria, ligada a la seguridad alimentaria. Por supuesto, cada producto tiene unos atributos nutricionales que pueden ser modificados en los procesos de transformación, transporte, etc. y que se asocian con determinadas cualidades sensoriales u organolépticas (sabor, olor, color, textura). El desarrollo tecnológico en las industrias agroalimentarias conforman nuevas características en la morfología y composición de los alimentos, facilitando la industrialización o comercialización del mismo. Existen también atributos relacionados con la salud que dan un valor añadido al alimento (alimentos dietéticos, enriquecidos, hipocalóricos, etc.). Otro valor añadido son los atributos éticos o emocionales, es decir, un conjunto de propiedades de importancia para el consumidor y que determina la adquisición o no del producto (ecológico, protección del medio ambiente, comercio justo, bienestar animal, etc.). Además, existen también unas características de uso que reúne los atributos relacionados con la fácil preparación o larga conservación u otras características que acaban siendo muy valoradas por determinados consumidores. 
Como se puede observar, la apreciación de la calidad de los alimentos y los atributos que los caracterizan no son inalterables y van evolucionando según se van desarrollado las estructuras socio-culturales y económicas de nuestra sociedad. Por otra parte, la importancia de los atributos descritos variará dependiendo de quién juzgue la calidad (productor, fabricante, consumidor, etc.) y a su vez la percepción de la calidad variará dependiendo de las características del consumidor (área geográfica, grupo social, económico o religioso, etc.), todo ello influenciado por técnicas de mercado que modifican gustos y necesidades del consumidor y alteran la percepción de la calidad, dando al producto atributos a veces intangibles.

La calidad del producto es así un concepto variable basado en atributos, y determinada por el grado de adecuación para usos y consumos. En concordancia con la Organización Internacional de Normalización (International Organization for Standardization, ISO), se puede definir la calidad como la capacidad de un producto o servicio de satisfacer las necesidades declaradas o implícitas del consumidor a través de sus características o propiedades.

Todos los eslabones de la cadena alimentaria contribuyen a la calidad y seguridad de los productos. En la producción primaria, primer eslabón de la cadena alimentaria, constituida por todos los profesionales y empresas que se dedican a la producción, cría o cultivo de los productos de la tierra, la ganadería, la caza y la pesca, se deben garantizar las condiciones higiénico-sanitarias, controlando contaminantes, plagas, enfermedades e infecciones en plantas y animales, evitando en todo momento impactos negativos en el medio ambiente. El siguiente eslabón lo constituyen las industrias agroalimentarias que fabrican, manipulan o transforman los productos, envasan, almacenan y transportan los productos alimenticios y que deben controlar la calidad y seguridad de los productos en todas las fases de su actividad. La comercialización y venta también es parte esencial para garantizar las cualidades óptimas en que llegará el producto al consumidor. El'último eslabón de la cadena lo constituyen los consumidores, responsables de demandar productos de calidad, con plenas garantías de inocuidad, que presenten información veraz y detallada de los alimentos, y responsables también de denunciar deficiencias, fraudes e incumplimientos de la normativa. Así también los atributos de calidad, antes descritos, son adquiridos en los diferentes eslabones de la cadena alimentaria.

La complejidad de la cadena alimentaria y los posibles riesgos sanitarios 
existentes resaltan la necesidad de implantar sistemas de control y gestión de la calidad para asegurarla y garantizarla. La calidad no sólo se controla o se evalúa sino que se obtiene e incorpora al producto en cada fase del proceso siendo necesaria su gestión y certificación.

\section{Concepto de calidad}

El concepto Calidad es un concepto dinámico que ha ido evolucionando a lo largo del tiempo. Así a principios del pasado siglo, el concepto Calidad estaba asociado a conseguir la uniformidad del producto, para ello se habían establecido una serie de estándares que recogían las características que se debían cumplir, y el papel de los profesionales de la calidad consistía en la inspección del producto en la etapa final del proceso productivo (control post-productivo), es decir, la calidad se comprueba.

Posteriormente, en 1920 y 1930, se establecen los primeros principios de la calidad, implementando el control estadístico en el proceso de verificación, realizando el control sobre muestras representativas de la producción y no sobre la producción completa, además también se realiza el control en etapas iniciales e intermedias del proceso; en esta época se empieza a hablar de control de la calidad.

El concepto Calidad ha seguido evolucionando, ya no sólo se busca la uniformidad del producto y se controla estadísticamente la producción, ahora se quiere asegurar o garantizar la calidad. Es necesario prevenir los errores y en ello están implicados todos los departamentos de la empresa e incluso los proveedores externos. El control se aplica a la totalidad del proceso, producto o servicio, lo que exige una planificación y evaluación de la calidad y un diseño de los programas de control. El aseguramiento de la calidad lleva consigo un conjunto de acciones planificadas y sistemáticas necesarias para proporcionar la confianza adecuada de que un producto satisfará los requisitos dados sobre la calidad. Además el aseguramiento no será completo si no se contemplan todas las necesidades del usuario. Esto implica la evaluación permanente de los factores que puedan influir y la verificación de todas las operaciones del proceso. Así el aseguramiento de la calidad se convierte en una herramienta de gestión para la empresa y un sistema de aumentar la confianza para el cliente.

La gestión de la calidad como recurso estratégico, considera la calidad como integrante global de la empresa y se gestiona como un factor decisivo para 
competir en el mercado, coordinando todos los recursos e intentando satisfacer las necesidades del cliente.

Actualmente se considera el concepto Calidad como un concepto integrador, calidad total, y con implicación no sólo en el control de la producción y la satisfacción del cliente, sino también en un compromiso con la sociedad, previniendo los posibles impactos medioambientales o con sus propios trabajadores, incentivando su formación e integración y previniendo los riesgos laborales.

El nuevo enfoque del concepto Calidad frente al enfoque tradicional se diferencia en que ya no se intenta cumplir únicamente los estándares fijados sino satisfacer todas las expectativas del cliente; no sólo se quiere detectar los errores sino prevenirlos. Con el nuevo enfoque la calidad es un valor añadido que optimiza la productividad y es rentabilizada en forma de competitividad, además ahora la responsabilidad no es de unos pocos sino de todos. Por otra parte, la calidad total ayuda a vender más y mejor, pues los clientes satisfechos repiten, el producto se diferencia de los "commodities", forzando a la vez a conocer mejor las necesidades del cliente, ayudando a su fidelización. Este aumento de la competitividad reduce costes y aumenta la capacidad empresarial.

\section{Sistemas de gestión de calidad}

La implantación de un sistema de calidad lleva implícita la aceptación de la cultura de la calidad, el convencimiento y mentalización de toda la organización de que la calidad es necesaria.

La organización debe establecer un sistema de la calidad apropiado a sus actividades (producción, distribución y servicios, etc.), sus características (ámbito territorial, tamaño, etc.) y a sus objetivos en materia de calidad.

El sistema de calidad debe ser diseñado, implantado y mantenido por la Dirección de la empresa. El sistema debe ser conocido y aceptado por toda la organización. Su principal característica será primar las actividades preventivas sobre las correctivas, siendo revisado periódicamente para estimular la mejora continuada de la calidad y satisfacer los requerimientos legales y normativos.

Se define por tanto el sistema de calidad como la estructura organizativa, los procedimientos, los procesos y los recursos necesarios para llevar a cabo la gestión de la calidad, y que tiene como finalidad alcanzar los objetivos definidos en la política de calidad.

Como se puede observar la documentación es uno de los puntales del 
sistema de calidad al garantizar la adecuación y el seguimiento de todas las actividades que se han llevado a cabo. Por otra parte, las auditorías, externas o internas, es un elemento esencial de evaluación del sistema y serán la base para la revisión del mismo, permitiendo la mejora continuada de la calidad.

La implantación de un sistema de calidad es una acción voluntaria y estratégica, por lo que antes de iniciar el proceso se deben tener en cuenta las siguientes consideraciones:

El momento de implantación. Antes de iniciar la implantación se debe estar seguro que es el momento adecuado para ello. Hay circunstancias que aconsejan aplazar la implantación, por ejemplo cuando a corto o medio plazo vaya a haber cambios en la estructura organizativa de la empresa, o tecnológicos, o haya previsto cambios en la estructura de terceros (clientes). Es relevante este aspecto porque el proceso de implantación, incluso en pequeñas empresas, puede ser superior a ocho meses y exigen, en general, importantes cambios para la adecuación de la organización.

El tipo de sistema que queremos y la motivación que genera su implantación. Será distinto el planteamiento si se quiere un sistema de calidad total en que el paso inicial será la implantación del sistema de control de calidad pero seguirá en el caso de las industrias alimentarias, un sistema de calidad higiénica (sistema APPCC), el sistema de gestión medioambiental y que también deberá hacer frente a los requisitos de seguridad y salud laboral mediante un plan de prevención de riesgos laborales. En organizaciones existentes que se ven obligadas o ven la necesidad de incorporar sistemas de calidad, debe valorarse la dificultad de seguir con el trabajo diario y asegurar su productividad, a la vez que paulatinamente se va incorporando la gestión y los principios propios de los sistemas de calidad.

Financiación de la implantación. La implantación del sistema de calidad requerirá un esfuerzo desde el punto de vista organizativo y por tanto económico. Se generan nuevos gastos en las acciones preliminares de la implantación (asesores, estudios de viabilidad, estudios de mercado, encuestas clientes, etc.), gastos de certificación, gastos tecnológicos, gastos de formación y gastos de personal (nuevos puestos de personal de calidad). Es necesario por tanto, un presupuesto de implantación 
realista que incorpore también los posibles ingresos derivados de la implantación (subvenciones, incrementos en las ventas, etc.).

Los gestores de calidad. La puesta en marcha de un sistema de calidad hace necesario contar con personal especializado para llevar a cabo estas tareas. Es deseable que sea un departamento autónomo, independiente de las otras áreas de la organización, el que implante objetivamente, haga funcionar y evalúe los sistemas de la calidad.

En general, es recomendable una implantación progresiva del sistema de calidad en las organizaciones existentes, mientras que en las nuevas organizaciones el diseño del sistema de calidad y su desarrollo va ligado al inicio de actividad de las mismas.

La calidad en una industria alimentaria tiene componentes peculiares que la diferencian de otros sectores. Los consumidores no sólo exigen productos que respondan a sus necesidades nutricionales, gustativas, etc. sino que también sean saludables y seguros. Como se ha visto en apartados anteriores, la calidad debe garantizar siempre la seguridad alimentaria.

\section{Modelo de calidad ISO 9000}

Aunque se reconocen varios sistemas de la calidad relacionados con normas internacionales, sin embargo la serie de normas de la Organización Internacional de Normalización (ISO), conocidas como ISO 9000, constituyen un conjunto de normas que proponen modelos genéricos para la implantación y el mantenimiento de sistemas de gestión de la calidad, suficientemente generales como para ser aplicados a cualquier tipo de organización, con independencia de sus características (ámbito empresarial, ubicación, tamaño, etc.). El modelo de calidad ISO 9000 se ha impuesto a nivel mundial como sistema de calidad en todos los sectores empresariales siendo ya identificado por los consumidores como símbolo de calidad y garantía. La familia de normas ISO (Tabla 1) apareció por primera vez en 1987, teniendo como base la normativa británica (British Retail Consortium, BRC) y extendiéndose de forma general a partir de 1994, siendo actualizadas en las posteriores versiones en armonización con otras normas. 
Tabla 1. Normas de la serie ISO 9000

\begin{tabular}{|c|c|c|}
\hline Norma ISO & Título & Norma UNE \\
\hline ISO 9000:2005 & $\begin{array}{c}\text { Sistema de gestión de la } \\
\text { calidad. Fundamentos } \\
\text { y vocabulario }\end{array}$ & $\begin{array}{c}\text { UNE-EN ISO 9000:2005 } \\
\text { (anula UNE-EN ISO } \\
9000: 2000)\end{array}$ \\
\hline ISO 9001:2008 & $\begin{array}{c}\text { Sistemas de gestión de la } \\
\text { calidad. Requisitos }\end{array}$ & $\begin{array}{c}\text { UNE-EN ISO 9001:2008 } \\
\text { (anula UNE-EN ISO } \\
9001: 2000)\end{array}$ \\
\hline ISO 9004:2000 & $\begin{array}{c}\text { Sistemas de gestión de la } \\
\text { calidad. Directrices para la } \\
\text { mejora del desempeño }\end{array}$ & $\begin{array}{c}\text { UNE-EN ISO 9004:2000 } \\
\text { (será anulada por } \\
\text { PNE-prEN ISO 9004) }\end{array}$ \\
\hline
\end{tabular}

La primera de ellas (UNE-EN ISO 9000:2005) recoge los conceptos, fundamentos, terminología y vocabulario relacionados con la gestión de la calidad. La segunda (UNE-EN ISO 9001:2008) especifica los requisitos de los sistemas de gestión de la calidad y fundamenta la base para su certificación. Por último, las directrices para la mejora continua hacia la excelencia son recogidas en la UNE-EN ISO 9004:2000.

Estas normas técnicas promueven la adopción de un enfoque basado en procesos que desarrolla, implementa y mejora la eficacia de un sistema de gestión de la calidad, para aumentar la satisfacción del cliente mediante el cumplimiento de sus requisitos. Un enfoque de este tipo enfatiza la importancia de:

La compresión y el cumplimiento de los requisitos.

La necesidad de considerar los procesos en términos que aporten valor. La obtención de resultados del desempeño y eficacia del proceso.

La mejora continua de los procesos con base en mediciones objetivas.

\section{Principios de la gestión de la calidad}

Las normas ISO 9000 se han basado en ocho principios de gestión de la calidad que recogen las mejores prácticas y que ya se han esbozado en el epígrafe anterior:

$1^{\text {er }}$ Principio. Organización orientada al cliente

Como puede observarse, el seguimiento de la satisfacción del cliente re- 
quiere la evaluación de la información relativa a la percepción del cliente acerca de si la organización ha cumplido sus requisitos.

La aplicación del principio de enfoque al cliente normalmente conduce a:

- Estudiar y comprender las necesidades y expectativas del cliente.

- Asegurarse de que los objetivos y metas de la organización están ligados a las necesidades y expectativas del cliente.

- Comunicar las necesidades y expectativas del cliente a toda la organización.

- Medir la satisfacción del cliente y actuar sobre los resultados.

- Gestionar de forma sistemática las relaciones con los clientes.

En definitiva, las organizaciones dependen de sus clientes y por lo tanto deberían comprender las necesidades actuales y futuras de los mismos, y satisfacer sus requisitos y esforzarse en exceder sus expectativas.

Los beneficios esperados serán un aumento de la cuota de mercado y de ingresos mediante una respuesta flexible y rápida al mismo, aumentado por otra parte la eficacia de la organización para aumentar la satisfacción del cliente

$2^{\circ}$ Principio. Liderazgo

Los líderes son personas que coordinan y equilibran los intereses de todos los grupos que de una u otra forma tienen interés en la organización. Los principales resultados previsibles serán:

- Considerar las necesidades de todas las partes interesadas.

- Establecer una clara visión del futuro de la organización.

- Establecer objetivos y metas desafiantes.

- Crear y mantener valores compartidos, imparcialidad y modelos éticos de comportamiento en todos los niveles de la organización.

- Crear confianza y eliminar temores.

- Proporcionar al personal los recursos necesarios, la formación y la libertad para actuar con responsabilidad y autoridad.

De esta forma las actividades se implementan y evalúan de una forma integrada, reduciendo la falta de comunicación entre los niveles de la organización.

$3^{\text {er }}$ Principio. Participación del personal

El personal es esencial en toda organización. El objetivo será comprender la 'importancia del papel desarrollado en la organización, identificando limitaciones, definiendo problemas y compartiendo conocimientos y experiencias, y 
asumiendo responsabilidades. Siendo su trabajo evaluado de acuerdo con los objetivos y metas fijados.

De esta forma se tendrá un personal motivado y comprometido con la organización, que promoverá innovación y creatividad, implicado en contribuir a la mejora continua.

$4^{\circ}$ Principio. Enfoque basado en procesos

El resultado deseado se alcanza más eficientemente cuando las actividades y los recursos relacionados se gestionan como un proceso, definiéndose éste como una secuencia de actividades que van añadiendo valor mientras se produce un determinado producto o servicio a partir de determinadas aportaciones. Todas las actividades de la organización se enmarcan dentro de procesos, que se identifican, gestionan y mejoran. Dentro de cada proceso se desarrolla el ciclo de gestión PHVA: planificar qué hacer y cómo, hacer lo planificado, verificar lo realizado y actuar. De esta forma:

- Se definen sistemáticamente las actividades necesarias para lograr el resultado deseado.

- Se establecen responsabilidades y obligaciones claras para la gestión de las actividades clave.

- Se analiza y mide la capacidad de las actividades clave.

- Se identifican las actividades clave dentro y entre las funciones de la organización.

- Se concretan los factores que mejorarán las actividades clave de la organización, como los recursos, métodos y materiales, etc.

$5^{\circ}$ Principio. Enfoque basado en un sistema para la gestión

Este enfoque permite identificar, entender y gestionar los procesos interrelacionados como un sistema que contribuye a la eficacia y eficiencia de una organización en la consecución de sus objetivos. Este principio debe conducir a:

- Estructurar un sistema para alcanzar los objetivos de la organización de la forma más eficaz y eficiente.

- Entender las interdependencias existentes entre los diferentes procesos del sistema.

- Estructurar los enfoques que armonizan e integran los procesos.

- Entender las capacidades organizativas y establecer las limitaciones de los recursos antes de actuar. 
- Definir y establecer como objetivo la forma en que deberían funcionar las actividades específicas dentro del sistema.

- Mejorar continuamente el sistema a través de la medición y la evaluación.

El principal beneficio es la coherencia, eficacia y eficiencia de la organización para conseguir los objetivos y la confianza por ello generada.

\section{$6^{\circ}$ Principio. Mejora continua}

La mejora continua en el desempeño global de la organización debe ser un objetivo permanente de ésta. Los principales resultados obtenidos con este enfoque serán el incremento de la competitividad al mejorar la estrategia y las capacidades organizativas y el desarrollo de mayor flexibilidad para reaccionar rápidamente frente a nuevos retos y situaciones. La mejora continua deber ser objetivo de todo el personal de la organización y debe potenciarse aportando medios de formación y estímulo y un seguimiento de la misma.

$7^{\circ}$ Principio. Enfoque basado en la toma de decisiones

Las decisiones deben ser eficaces por lo que deben basarse en el análisis de los datos y la información veraz y relevante, incluyendo la percepción de todos los grupos de interés.

\section{$8^{\circ}$ Principio. Relaciones con el proveedor}

Las organizaciones se enmarcan dentro de una cadena de proceso clientesproveedores. Las organizaciones y sus proveedores son interdependientes, y una relación mutuamente beneficiosa aumenta la capacidad de ambos para crear valor. Las principales ventajas de establecer relaciones con el proveedor es la mejora en la flexibilidad y rapidez de respuesta en forma conjunta a un mercado cambiante o a las necesidades y expectativas del cliente, así como la optimización de costos y recursos.

Los principios enunciados deben permitir que el sistema de calidad demuestre que la organización es capaz de suministrar un producto o servicio que de manera consistente, cumpla con los requisitos de los clientes y las reglamentaciones correspondientes y además, lograr la satisfacción del cliente mediante la aplicación efectiva del sistema, incluyendo la prevención de fallos y el proceso de mejora continua. 


\section{GESTIÓN DE LA CALIDAD EN LA INDUSTRIA AGROALIMENTARIA}

Las industrias agroalimentarias necesitan conseguir la homogeneidad, calidad y seguridad en el producto final, pero también reconocen la necesidad de demostrar su capacidad para identificar y controlar los peligros relacionados con la seguridad de los alimentos.

Estas industrias han ido implementando progresivamente esquemas globales de garantía y de gestión de la calidad. Así, modelos de calidad como ISO 9000 e ISO 14000, que engloban todos los aspectos de la calidad, inicialmente desarrollados en otros sectores y que hemos descrito anteriormente han ido incrementando su implantación en el panorama industrial español. Sin embargo, existe cierta tendencia a separar la calidad higiénico-sanitaria de otros aspectos de la calidad.

Tradicionalmente, la gestión de la seguridad de los alimentos ha sido mayoritariamente competencia exclusiva de los Estados, con el objetivo primordial de proteger la salud pública, y orientados en las cuestiones normativas por los organismos internacionales que se ocupan de los diversos aspectos de la inocuidad alimentaria, en particular la Comisión del Codex Alimentarius. Sin embargo, en los últimos años, el sector privado ha desempeñado un gran papel en la gestión de la inocuidad de los alimentos, especialmente en el mundo desarrollado. De conformidad con este enfoque de la gestión de la inocuidad de los alimentos, incumbe a la industria ejecutar programas de garantía de la calidad y la inocuidad de los alimentos, orientados a prevenir los problemas de seguridad alimentaria en todas las etapas de la cadena de suministros. De esta forma se logra una gestión de la inocuidad de los alimentos más eficaz, compartiendo la responsabilidad para garantizarla entre todos los implicados en la cadena alimentaria.

Las industrias agroalimentarias iniciaron el aseguramiento de la calidad desarrollando buenas prácticas de fabricación (BPF), recogidas por el Codex Alimentarius en el Código de Buenas Prácticas Higiénicas, y que constituyen un conjunto de normas mínimas para la obtención de un producto inocuo y saludable. La implantación de las BPF es una herramienta básica e imprescindible para la obtención de productos seguros para el consumo humano. Inciden en el control del personal involucrado, las instalaciones de fabricación y el producto fabricado, su higiene y manipulación.

Posteriormente, se desarrollaron los sistemas de análisis de peligros y puntos críticos (APPCC) que gestionan fundamentalmente los riesgos higiénicos-sani- 
tarios, y que desarrollaremos más extensamente en el epígrafe posterior. Se utilizan para controlar y detectar cualquier peligro que afecte a la inocuidad de los alimentos y que pueda evitarse, eliminarse o reducirse a niveles aceptables. Esta metodología no debe ni puede reemplazar a las BPF. Sin embargo su aplicación es el primer paso para lograr la implantación con éxito de las BPF.

Aunque existen otros estándares de seguridad y calidad alimentaria, por ejemplo, Eurep-gap, British Retail Consortium (BRC), UNE 155000, International Food Standard (IFS), Safe Quality food (SQF), en la actualidad la industria agroalimentaria cuenta con una norma específica sobre los sistemas de gestión de inocuidad alimentaria, la norma ISO 22000:2005, respaldada por el consenso internacional, y que armoniza los requisitos para implementar la seguridad en la cadena alimentaria. En el desarrollo de la norma han participado expertos de la industria alimentaria, representantes de organizaciones internacionales especializadas, en cooperación con la Comisión del Codex Alimentarius de FAO/OMS.

La ISO 22000:2005 es un modelo único de sistema de gestión de seguridad alimentaria que cubre todos los requisitos y actividades de la cadena alimentaria, desde el productor primario al almacenaje y los servicios externos a las empresas. Incorpora los principios de APPCC y otros programas de prerequisitos de higiene alimentaria, siendo compatibles con las industrias que tienen implementado el sistema ISO 9000. Este sistema de gestión de la inocuidad alimentaria (SGIA) es aplicable a todas las organizaciones, independientemente del tamaño, abarcando aspectos como la comunicación interactiva, el sistema de gestión y el control de riesgos.

Sus aspectos más sobresalientes son:

- El compromiso formal de la Dirección de la organización para la producción de alimentos inocuos.

- La satisfacción de los requisitos de los consumidores y legales aplicables.

- Se involucra a todos los que conforman la cadena alimentaria.

- Se establece una comunicación interactiva entre todos los eslabones de la cadena e internamente dentro de la organización.

- Hay una actualización continua y demostrable del SGIA.

- Hay un tratamiento de contingencias.

- La trazabilidad es comprobable: hacia atrás, interna o de proceso, hacia delante. 
- Hay control de documentos y registros.

- Incluye específicamente a las PYMES.

- Es un sistema de gestión que abarca todas las estructuras de la organización.

En la tabla siguiente (Tabla 2) se comparan los diferentes aspectos de la norma ISO 22000:2005 con otros estándares de seguridad alimentaria.

Tabla 2. Comparación de la ISO 22000 con otros estándares (European Quality Assurance, EQA).

\begin{tabular}{|c|c|c|c|c|}
\hline ISO 22000 & ISO 9001 & APPCC & BRC & IFS \\
\hline $\begin{array}{l}\text { Sistema de } \\
\text { Gestión de } \\
\text { Inocuidad } \\
\text { Alimentaria }\end{array}$ & $\begin{array}{l}\text { Sistema de } \\
\text { Gestión } \\
\text { de Calidad }\end{array}$ & $\begin{array}{l}\text { Principio } 7 \\
\text { (Documentación } \\
\text { y registro) }\end{array}$ & $\begin{array}{l}\text { Sistema de } \\
\text { Gestión } \\
\text { de Calidad }\end{array}$ & $\begin{array}{l}\text { Sistema de } \\
\text { Gestión } \\
\text { de Calidad }\end{array}$ \\
\hline $\begin{array}{l}\text { Responsabilidad } \\
\text { de la Dirección }\end{array}$ & $\begin{array}{l}\text { Responsabilidad } \\
\text { de la Dirección }\end{array}$ & & $\begin{array}{l}\text { Responsabilidad } \\
\text { de la Dirección }\end{array}$ & $\begin{array}{l}\text { Responsabilidad } \\
\text { de la Dirección }\end{array}$ \\
\hline $\begin{array}{l}\text { Gestión } \\
\text { de recursos }\end{array}$ & $\begin{array}{l}\text { Gestión de } \\
\text { recursos }\end{array}$ & $\begin{array}{l}\text { Establecimiento } \\
\text { del equipo APPCC }\end{array}$ & $\begin{array}{l}\text { Gestión de } \\
\text { recursos }\end{array}$ & $\begin{array}{l}\text { Gestión de } \\
\text { recursos }\end{array}$ \\
\hline $\begin{array}{l}\text { Planificación y } \\
\text { realización de } \\
\text { productos seguros }\end{array}$ & $\begin{array}{l}\text { Realización } \\
\text { del producto }\end{array}$ & $\begin{array}{l}\text { Principio 1-3 } \\
\text { (lista de peligros, } \\
\text { determinación de } \\
\text { PCC y límites críticos) }\end{array}$ & $\begin{array}{l}\text { Control del } \\
\text { producto }\end{array}$ & $\begin{array}{l}\text { Realización } \\
\text { del producto }\end{array}$ \\
\hline $\begin{array}{l}\text { Validación, } \\
\text { verificación y } \\
\text { mejora del SGIA }\end{array}$ & $\begin{array}{l}\text { Medición, análisis } \\
\text { y mejora }\end{array}$ & $\begin{array}{l}\text { Principio 4-6 } \\
\text { (seguimiento, acciones } \\
\text { correctivas, verificación) }\end{array}$ & $\begin{array}{l}\text { Control del } \\
\text { producto y } \\
\text { proceso }\end{array}$ & $\begin{array}{l}\text { Medición, análisis } \\
\text { y mejora }\end{array}$ \\
\hline
\end{tabular}

